\title{
High-intensity activity according to playing position with different team formations in soccer
}

\author{
Javier J. Vilamitjana ${ }^{1}$, Gabriel Heinze ${ }^{2}$, Pablo Verde ${ }^{3}$, and Julio Calleja-González ${ }^{4}$ \\ ${ }^{1}$ Soccer Research Group, Friends Club-CDA, Buenos Aires, Argentina; ${ }^{2}$ Sports and Cultural Association of Crespo, Crespo, Argentina; \\ ${ }^{3}$ Coordination Centre of Clinical Trials, University of Dusseldorf, Dusseldorf, Germany; and ${ }^{4}$ Physical Activity and Sports Sciences, \\ University of the Basque Country - UPV/EHU, Leioa, Spain
}

\begin{abstract}
Background: A tactical factor such as playing formation seems to be another influencing factor in the physical performance of elite soccer players during the match. Some researchers have suggested that distances covered during high-intensity running in matches are valid measures of physical performance. They concluded that players covered greater distances of high-intensity activities during some team formations in comparison to others. Objective: The aim of this study was to examine high-intensity patterns of professional soccer players in relation to the positional role with two different playing formations. Methods: Match data were collected during official games systematically playing in 1-3-4-3 and 1-4-2-1-3 formations. Nineteen professional players (age $24.7 \pm 4.8$ years, body mass $74.5 \pm 6.2 \mathrm{~kg}$, height $176.3 \pm 5.3 \mathrm{~cm}$, percentage of body fat $9.7 \pm 2.5 \%$ ) were classified into five positional roles: central defender, wide defender, midfielder, wing and forward. Match performance variables included moderate-intensity running $(14.9-19.8 \mathrm{~km} / \mathrm{h})$, high-speed running (19.9-25.2 km/h) and sprinting (> $25.2 \mathrm{~km} / \mathrm{h})$. The number of runs (\#HSR, \#SPR) and metabolic rates as HILR $([\mathrm{MIR}+\mathrm{HSR}+\mathrm{SPR}] / \mathrm{min})$ and HSSL ([HSR + SPR $] / \mathrm{min})$ were determined. Results: The statistical analysis revealed that \#SPR $(p=.045)$, HILR $(p=.022)$ and HSSL $(p=.019)$ were higher in 1-4-2-1-3 than 1-3-4-3 formation. According to the playing position, significant differences were found in HILR $(p=.045)$ and HSSL $(p=.028)$ for forwards during 1-4-2-1-3 and midfielders amounted more HILR than others in that team formation $(p=.047)$. Additionally, wings amounted significantly higher \#HSR $(p=.011)$ and \#SPR $(p=.010)$ in 1-4-2-1-3, as long as forwards was the other position with more \#SPR during that formation $(p=.023)$. Conclusions: The players performed more high-intensity patterns in 1-4-2-1-3. Attackers and midfielders were the playing positions that held the most statistical differences comparing both team formations. These findings reveal that playing formation seems to be another potential factor of influence with respect to the physical performance of elite players if we consider their high-intensity profile in particular.
\end{abstract}

Keywords: professional soccer player, tactical formation, positional role, match demands analysis, high-intensity patterns, sprinting performance

\section{Introduction}

Although physical and technical indicators can be seen as individual parameters of match-play, success in soccer is a culmination of suitable tactics completed with the appropriate level of physical and technical performance (Bush et al., 2015). Analyzing each of these mentioned indicators in isolation restricts the context, understanding and application of the findings. Therefore, additional research should adopt an integrated approach that analyzes physical, technical and tactical indicators of various playing positions in order to understand the overall development of soccer match-play (Bush et al., 2015). Among these, some researchers have suggested that distances covered during high-intensity running (HIR; > $14.9 \mathrm{~km} / \mathrm{h}$ ) in matches are valid in order to measure the physical performance in soccer-related to training status (Krustrup et al., 2003, 2005). However, research evidence typically uses a reductionist approach whereby match running performance is examined in detail without any integration of different factors (score line, level of opposition, tactical systems, game location), and this ultimately leads to a one-dimensional insight into match performance. On the other hand, previous findings demonstrated that high-speed running (HSR; > $19.9 \mathrm{~km} / \mathrm{h}$ ) was a distinguishing characteristic among players in dissimilar playing positions (Bradley et al., 2009), and tactical factor such as the playing formation seems to be another influential factor on the physical performance of elite players. For instance, Aquino et al. (2017) compared the differences between 1-43-3 and 1-4-4-2 formations in one reference team during 48 matches from the Brazilian national league. The results showed that players covered greater distances and increased their frequency of high-intensity activities during 1-4-3-3 formation play compared to 1-4-4-2 formations. In another study, 70 matches from the English FA Premier League team formations were analysed when they played against a

\footnotetext{
$\triangle$ Corresponding author: Javier J. Vilamitjana, e-mail vilamitjana@yahoo.com, ORCID ${ }^{\circledR}$ record https://orcid.org/0000-0002-8752-1276 Article history: Received August 6 2020, Accepted February 9 2021, Published March 292021

Copyright: (c) 2021 The Author(s). Published by Palacký University Olomouc. This is an open access article distributed under the terms of the Creative Commons Attribution License (https://creativecommons.org/licenses/by/4.0/), which permits unrestricted use, distribution, and reproduction in any medium, provided the original author and source are credited. This license does not cover any third-party material that may appear with permission in the article.
} 
1-4-4-2 defensive tactic (Bradley et al., 2011). The authors concluded that no differences were found for the overall running performance of players playing in several team formations (1-4-3-3 and 1-4-5-1), but distances covered at different intensities varied substantially for playing positions across formations: Attackers in a 4-3-3 formation covered $28-32 \%$ more HIR than attackers in 1-4-5-1 and 1-4-4-2 formations, and defenders in a 1-4-4-2 ran $11 \%$ more distance in HIR than those in a 1-4-5-1 formation.

Tierney et al. (2016) evaluated the physical demands in elite level football players of this particular 1-4-2-3-1 formation (professional players with at least 2 years' playing experience of elite level; $20 \pm 3$ years). Authors determined that high-speed running values in 1-4-2-3-1 were lower compared to $1-3-5-2$ (538 vs. $642 \mathrm{~m}$ ) but similar to 1-43-3 and 1-3-4-3 formations (514 vs. $551 \mathrm{~m}$, respectively). In addition, central midfielders in 1-4-3-3 covered greater total distance compared to 1-4-4-2 (14\% more), and forwards in 1-3-5-2 covered greater high-speed running (> $19.8 \mathrm{~km} / \mathrm{h}$ ) than in $1-4-2-3-1$ (45\% more).

However, based on an in-depth examination of high intensity running across different tactical formations (e.g., 1-3-4-3 or 1-4-2-1-3) could provide insight into positionspecific changes during the game and supply a tool for optimal training preparation. Furthermore, to the best of these authors' knowledge, no previous study of professional soccer players has investigated high-intensity activity patterns in relation to the positional role with these playing formations at the highest competitive level in South America.

Therefore, the aim of the present study was to examine the high-intensity activity patterns of professional soccer players in relation to the positional role with two playing formations (1-3-4-3 vs. 1-4-2-1-3) during official soccer games.

\section{Methods}

\section{Participants}

Nineteen professional soccer players (age $24.7 \pm 4.8$ years, body mass $74.5 \pm 6.2 \mathrm{~kg}$, height $176.3 \pm 5.3 \mathrm{~cm}$, percentage of body fat $9.7 \pm 2.5 \%$ ) participated in this study; all of them were playing for an elite Argentinean team, competing in the Second League during 2016-17 season. The players were grouped by their position on the pitch: central defenders $(n=4)$, wide defenders $(n=3)$, midfielders $(n=5)$, wingers $(n=5)$, and forwards $(n=2)$. Goalkeepers were excluded from the study, as they did not participate in the same physical training program as the rest of the squad.

Before the start of the season, the players were evaluated using FIFA's medical protocol. None of them presented any ailments, pathologies or injuries and no medical prescriptions were issued. The body composition and body fat mass determination were assessed from skinfolds measurements (Jackson \& Pollock, 1978).

All the participants were informed of the research objectives and volunteered to participate in the study, which in no way disrupted their scheduled training. The study protocol was approved by the local Institutional Review Board and drafted in accordance with the Declaration of Helsinki.

\section{Procedure}

The data for each match were gathered during the 2016-17 season. In terms of the two tactical formations used by the coach in the course of the competition, $52 \%$ of the games (23) were played using the 1-3-4-3 formation (goalkeeper, three central defenders, two wide defenders, two midfielders, one forward and two wingers) and 48\% (21 games) using the 1-4-2-1-3 formation (goalkeeper, two central defenders, two wide defenders, three midfielders, one forward and two wingers). All the matches were played on natural grass football pitches with standard dimensions.

During the season, each player's GPS and heart rate were monitored 16 times (four observations of each playing formation were selected in each semester of the season: August-December, March-July). During the match, the total distance covered for each individual player was determined. Their maximal and mean heart rate (HR; beats per $\mathrm{min}$ ) were also recorded. The only players which were taking into consideration where those who completed the total time of each game under normal conditions and also held the same role on the pitch.

In each competitive micro-cycle, the players performed five sessions with the ball (one day of possession games, three days of tactical-position games and one day of "stop balls"), each session of which lasted 45 min net (average). There were one to two weekly strength sessions plus the match (the average total distance covered for all activities per micro-cycle was $31.6 \mathrm{~km}$ ). There were no differences in weekly methodological structure when the team played with different tactical systems.

At the end of the season the team finished in the first position (games won: 25, drew: 13, lost: 6).

\section{Instruments and measurements}

All the physical and physiological parameters were assessed using portable $10-\mathrm{Hz}$ GPS devices (Zephyr, Qstarz Q818XT, Annapolis, MD, USA) and analyzed using Zephyr BioHarness v4.1.4. HR responses were recorded continuously using Zephyr BioHarness (previously validated by Brooks et al., 2013; Kim et al., 2013). To limit inter-unit error, each player wore the same device throughout the course of the season. GPS devices were activated 15 min prior to the start of the match, in accordance with the manufacturer's instructions (GPS accuracy: $<3$ m circular error probability [50\%] without selective availability [horizontal], typical differential GPS [Wide Area Augmentation System, Euro Geostationary Navigation Overlay Service, Satellite Augmentation System]: 2.5).

The following speed intensities were used to categorize motion: moderate-intensity running (14.9-19.8 km/h), high-speed running (19.9-25.2 km/h), and sprint running $(>25.2 \mathrm{~km} / \mathrm{h}$ ). The number of runs and the distance covered at each speed intensity were measured. The speed thresholds were equal to those reported previously by $\mathrm{Di}$ Salvo $(2009,2013)$. The six study variables were obtained from these parameters:

- high-intensity load rate (HILR): quotient between distance covered at moderate intensity, high-speed running 
and sprint by the amount of time of each activity in metres per minute,

- high speed running/sprints load rate (HSSL): quotient between distance covered at high-speed running and sprint by the amount of time of each activity in metres per minute,

- number of runs between 19.9 and $25.2 \mathrm{~km} / \mathrm{h}$ (\#HSR),

- number of sprints above $25.2 \mathrm{~km} / \mathrm{h}$ (\#SPR),

- average heart rate obtained during the observed period in beats per minute (Mean HR),

- maximal heart rate obtained during the observed period in beats per minute (Maximal HR).

The HILR and HSSL metabolic rates were previously reported by Vilamitjana et al. (2020).

\section{Statistical analysis}

A preliminary exploratory analysis was carried out followed by a normality (Shapiro-Wilk) and homogeneity test (Levene). Descriptive statistics measurements such as mean and standard deviation were calculated for each position and formation. A linear mixed-effects model was used to determine position and formation effects. For each response variable, the fixed effects were the formation and the position. The player was considered to be a random effect. This model makes it possible to calculate the formation and position effect separately, considering an autocorrelation between observations made about the same players (Gelman \& Hill, 2007). The model was adjusted using the restricted maximum likelihood method (McGilchrist \& Yau, 2007). The results include the table with the estimation of the model's parameters, standard errors and $p$-values. Statistically significant differences were reported at a level of $5 \%$. The statistical calculations were performed with the R statistical software (Version 3.4.3; R Foundation for Statistical Computing, Vienna, Austria). The statistical report was prepared with the KNITR statistical package which makes it possible to replicate all aspects of the analysis ( $\mathrm{R}$ package version 1.30, https://yihui.org/knitr).

\section{Results}

The means $( \pm S D)$ of the metrics for the different playing formation are shown in Table 1 . The statistical analysis revealed that HILR (21.6 vs. $19.9 \mathrm{~m} / \mathrm{min}, p=.022$ ), HSSL (7.8 vs. $6.7 \mathrm{~m} / \mathrm{min}, p=.019)$ and \#SPR (7.2 vs. 6.2 runs, $p=.045)$ obtained by all examined players were higher in 1-4-2-1-3 than 1-3-4-3 formation (Table 1).

In relation to positional roles, statistically significant differences were found in HILR and HSSL for forwards during 1-4-2-1-3 formation (23.6 vs. $17.9 \mathrm{~m} / \mathrm{min}, p=.045$ and 10.7 vs. $6.4 \mathrm{~m} / \mathrm{min}, p=.028$, respectively) in comparison with 1-3-4-3 formation (Table 2). Midfielders were the only other position with higher HILR values in that team formation (20.1 vs. $18.1 \mathrm{~m} / \mathrm{min}, p=.047$; Table 2).

Regarding numbers of running actions, the wings were the players with the greatest number of \#HIR and \#SPR in

Table 1 Means $\pm S D$ s of the metrics for the different playing formations

\begin{tabular}{lcc}
\hline & \multicolumn{2}{c}{ Playing formation } \\
\cline { 2 - 3 } Variable & $1-3-4-3$ & $1-4-2-1-3$ \\
\hline HILR (m/min) & $19.9 \pm 4.4$ & $21.6 \pm 3.3^{*}$ \\
HSSL (m/min) & $6.7 \pm 2.6$ & $7.8 \pm 2.6^{*}$ \\
\#HSR (runs) & $15.7 \pm 3.1$ & $15.7 \pm 2.3$ \\
\#SPR (runs) & $6.2 \pm 3.2$ & $7.2 \pm 3.3^{*}$ \\
Mean HR (beats/min) & $193.3 \pm 12.7$ & $193.7 \pm 6.6$ \\
Maximal HR (beats/min) & $170.3 \pm 7.5$ & $171.0 \pm 8.2$ \\
\hline
\end{tabular}

Note. $\mathrm{HILR}=$ high intensity load rate; $\mathrm{HSSL}=$ high speed running/sprints load rate; $\# H S R=$ number of high speed runs; \#SPR = number of sprints; $\mathrm{HR}=$ heart rate. ${ }^{*} p<.05$.

Table 2 Means $\pm S D$ s of the metrics for the different playing formations by positional roles

\begin{tabular}{|c|c|c|c|c|c|}
\hline Variable & Central defenders & Wide defenders & Midfielders & Wings & Forwards \\
\hline \multicolumn{6}{|c|}{ HILR (m/min) } \\
\hline $1-3-4-3$ & $15.5 \pm 1.3$ & $23.8 \pm 4.7$ & $18.1 \pm 3.7$ & $23.5 \pm 2.0$ & $17.9 \pm 2.0$ \\
\hline $1-4-2-1-3$ & $18.0 \pm 1.3$ & $23.7 \pm 2.5$ & $20.1 \pm 3.1^{*}$ & $23.9 \pm 1.7$ & $23.6 \pm 0.7^{*}$ \\
\hline \multicolumn{6}{|c|}{$\mathrm{HSSL}(\mathrm{m} / \mathrm{min})$} \\
\hline $1-3-4-3$ & $4.4 \pm 0.9$ & $8.1 \pm 2.0$ & $5.0 \pm 1.3$ & $9.4 \pm 2.0$ & $6.4 \pm 1.0$ \\
\hline $1-4-2-1-3$ & $6.0 \pm 1.4$ & $8.7 \pm 1.1$ & $5.2 \pm 1.2$ & $10.1 \pm 1.2$ & $10.7 \pm 1.9 *$ \\
\hline \multicolumn{6}{|l|}{ \#HIR (runs) } \\
\hline $1-3-4-3$ & $19.8 \pm 4.3$ & $25.0 \pm 2.7$ & $17.0 \pm 4.9$ & $20.2 \pm 0.8$ & $28.0 \pm 5.1$ \\
\hline $1-4-2-1-3$ & $19.0 \pm 1.8$ & $25.1 \pm 2.7$ & $18.1 \pm 3.4$ & $25.7 \pm 1.8^{*}$ & $26.9 \pm 2.7$ \\
\hline \multicolumn{6}{|l|}{ \#SPR (runs) } \\
\hline $1-3-4-3$ & $4.2 \pm 1.1$ & $7.2 \pm 1.7$ & $3.7 \pm 2.4$ & $5.4 \pm 0.8$ & $9.1 \pm 3.7$ \\
\hline $1-4-2-1-3$ & $5.1 \pm 0.7$ & $7.7 \pm 1.2$ & $3.9 \pm 1.3$ & $8.6 \pm 1.0^{*}$ & $11.1 \pm 2.5^{*}$ \\
\hline \multicolumn{6}{|c|}{ Mean HR (beats/min) } \\
\hline $1-3-4-3$ & $167.4 \pm 9.4$ & $176.1 \pm 6.1$ & $170.9 \pm 6.5$ & $160.1 \pm 12.7$ & $172.1 \pm 3.3$ \\
\hline $1-4-2-1-3$ & $169.8 \pm 6.3$ & $174.1 \pm 7.9$ & $174.0 \pm 7.2$ & $160.6 \pm 14.1$ & $171.4 \pm 3.0$ \\
\hline \multicolumn{6}{|c|}{ Maximal HR (beats/min) } \\
\hline $1-3-4-3$ & $190.4 \pm 8.8$ & $196.7 \pm 3.4$ & $193.7 \pm 5.4$ & $185.2 \pm 4.4$ & $194.9 \pm 4.0$ \\
\hline $1-4-2-1-3$ & $191.6 \pm 7.5$ & $195.4 \pm 3.7$ & $195.6 \pm 3.9$ & $182.2 \pm 8.4$ & $197.2 \pm 3.9$ \\
\hline
\end{tabular}

Note. $\mathrm{HILR}=$ high intensity load rate; $\mathrm{HSSL}=$ high speed running/sprints load rate; $\# \mathrm{HIR}=$ number of high intensity runs; \#SPR = number of sprints; $\mathrm{HR}=$ heart rate. ${ }^{*} p<.05$. 
the 1-4-2-1-3 formation (25.7 vs. 20.2 runs, $p=.011$ and 8.6 vs. 5.4 runs, $p=.010$, respectively) and the forwards were the other position with more \#SPR runs during that playing formation (11.1 vs. 9.1, $p=.023$; Table 2).

\section{Discussion}

The aim of the present study was to examine the highintensity activity patterns of professional soccer players in relation to the positional role with different playing formations (1-3-4-3 vs. 1-4-2-1-3) during official soccer games. This study revealed that HILR (distance covered in high intensity running per minute) and HSSL (distance covered in very high intensity per minute) were significantly higher in 1-4-2-1-3.

In relation to positional roles, statistically significant differences were found in HILR and HSSL for forwards and midfielders in HILR during 1-4-2-1-3 formation. Tactical explanations can be put forth to explain these differences: in 1-4-2-1-3 formation, midfielders and attackers probably assume a higher defensive activity without ball possession, with more contribution during the pressing. There are studies that have examined high-intensity activity, where attackers in a 1-4-3-3 (similar formation as 1-4-2-1-3) performed $-30 \%$ higher HIR than attackers in 1-4-4-2 and 1-4-5-1 formation (Bradley et al., 2011). In another study, the authors concluded that forwards in the 1-4-3-3 formation often assume defensive function without ball possession, in comparison with 1-4-4-2 formation, contributing to the midfield sector, also possibly resulting in greater physical output (Aquino et al., 2017). Moreover, Tierney et al. (2016) observed significantly higher high-speed running values in forwards (> 45\%) during 1-3-5-2 compared to 1-4-2-3-1 formation (U-19 and U-21 professional players).

Concerning to \#HSR, no differences were found between both tactical formations. Otherwise, the results from the present study confirm that wings were the positional role with more runs accumulated during 1-4-2-1-3 formation. Perhaps, this might be attributed to the physical capacity of the players and variation in task-specific requirements for each position (Bangsbo \& Peitersen, 2000; Mohr et al., 2003). Another tactical explanation can explain this phenomenon: wings in the 1-4-2-1-3 formation possibly assume a higher defensive function like "pressing" and other actions without the ball, resulting in greater physical output (Aquino et al., 2017). Further analysis from literature concluded that the number of high-intensity runs $(\geq 14.4 \mathrm{~km} / \mathrm{h}$ ) across playing positions was higher in attackers (forwards and wings) during 1-4-3-3 compared with a 1-4-5-1 or 1-4-4-2 (Bradley et al., 2011).

In our study, \#SPR were higher in 1-4-2-1-3 format compared to 1-3-4-3, with wings and forwards performing a significantly higher amount of \#SPR in that playing formation. The system of pressure could be an explanation: during 1-4-3-3 (similar as 1-4-2-1-3), attackers might be expected to pressure the back line and close down on opponents when unfavorably outnumbered, resulting in a greater performance at higher velocities (Bradley et al., 2011). Researchers have focused their attention on sprinting activities during matches in different international leagues and attackers seem to be the players who sprint the most of all positional roles (Andrzejewski et al., 2015; Mohr et al., 2003).

Finally, previous studies have demonstrated that HR monitoring is an interesting tool to evaluate the internal load in soccer games (Alexandre et al., 2012), but few studies have undertaken HR responses when different team formations are compared. In the present study, no significant differences were found in mean and maximal HR for all examined players when both formations are compared. We did not consider the influence of environmental conditions (temperature, humidity, wind speed) or the time of the day (hormonal circadian biorhythm), which are factors that have the capacity to alter the HR responses during the games (Alexandre et al., 2012). Soccer is a multifactorial activity and HR monitoring is only one of the different tools (at our disposal) to examine the physiologic and internal load. On the other hand, our study has another limitation: only one team was considered. Further studies will analyze the interaction of different positions from several teams, in a specific tactical system and the combination of HR with other physiological parameters (as metabolic thresholds or rated perceived exertion values), to measure the workload in relation to positive or negative training outcomes (Jaspers et al., 2017). This diagnosis could provide important information to establish goals and strategic projections for physical preparation, match, and recovery (Alexandre et al., 2012).

\section{Conclusions}

The most salient finding resulting from this study was that players displayed metabolic rates and performed more sprints in 1-4-2-1-3 when compared with 1-3-4-3 formation. Attackers and midfielders were the playing positions that had more statistical differences when both tactics were taking into consideration. These findings reveal that the playing formation seems to be another influential factor in the physical performance of elite players.

It is necessary to bring out the information that GPS metrics provide, in order to improve our understanding of how it can be best employed by coaches and fitness trainers. It appears that playing in different positions and across different formations impose different physical demands. Monitoring metabolic rates as HILR and HSSL, or the number of actions as \#HIR and \#SPR are relevant to be taken into account when high-intensity patterns are assessed. This could be used to increase training specificity by providing information for training prescription and periodization according to individual needs and competition demands.

\section{Acknowledgments}

The authors would like to thank all the players at the club for their participation in this study and also our colleagues Mariano Toedtli, Julio Vaccari. and Diego Navone who helped to carry it out. 


\section{Conflict of interest}

The authors report no conflict of interest.

\section{References}

Alexandre, D., Da Silva, C. D., Hill-Haas, S., Wong, D. P., Natali, A. J., De Lima, J. R. P., Filho, M. G. B. B., Marins, J. J. C. B., García, E. S., \& Karim, C. (2012). Heart rate monitoring in soccer: Interest and limits during competitive match play and training, practical application. Journal of Strength and Conditioning Research, 26(10), 2890-2906. https://doi.org/10.1519/JSC.0b013e3182429ac7

Andrzejewski, M., Chmura, J., Pluta, B., \& Konarski, J. (2015). Sprinting activities and distance covered by top level Europa League soccer players. International Journal of Sports Science \& Coaching, 10(1), 39-50. https://doi. org/10.1260/1747-9541.10.1.39

Aquino, R., Palucci Vieira, L. H., Carling, C., Martins, G. G. H. M., Alves, I. S., \& Puggina, E. F. (2017). Effects of competitive standard, team formation and playing position on match running performance of Brazilian professional soccer players. International Journal of Performance Analysis in Sports, 17(5), 695-705. https://doi.org/10.1080/24748668.2017.1384976

Bangsbo, J., \& Peitersen, B. (2000). Soccer systems and strategies. Human Kinetics.

Bradley, P. S., Carling, C., Archer, D., Roberts, J., Dodds, A., Di Mascio, M., Paul, D. Gomez Diaz, A., Peart, D., \& Krustrup, P. (2011). The effect of playing formation on high intensity running and technical profiles in English FA Premier League soccer matches. Journal of Sports Sciences, 29(8), 821-830. https://doi.org/10.1 $\underline{080 / 02640414.2011 .561868}$

Bradley, P. S., Sheldon, W., Wooster, B., Olsen, P., Boanas, P., \& Krustrup, P. (2009). High-intensity running in English FA Premier League soccer matches. Journal of Sports Sciences, 27(2), 159-168. https://doi.org/10.1080/02640410802512775

Brooks, K. A., Carter, J. G., \& Dawes, J. J. (2013). Comparison of VO2 measurement obtained by a physiological monitoring device and the Cosmed Quark CPET. Journal of Novel Physiotherapies, 3, Article 126. https://doi. org/10.4172/2165-7025.1000126

Bush, M., Barnes, C., Archer, D. T., Hogg, B., \& Bradley, P. S. (2015) Evolution of match performance parameters for various playing positions in the English Premier League. Human Movement Science, 39, 1-11. https://doi.org/10.1016/i. humov.2014.10.003
Di Salvo, V., Gregson, W., Atkinson, G., Tordoff, P., \& Drust, B. (2009). Analysis of high intensity activity in Premier League soccer. International Journal of Sports Medicine, 30(3), 205-212. https://doi.org/10.1055/s-0028-1105950

Di Salvo, V., Pigozzi, F., González-Haro, C., Laughlin, M. S., \& De Witt, K. (2013). Match performance comparison in top English soccer leagues. International Journal of Sports Medicine, 34(6), 526-532. https://doi.org/10.1055/s-0032-1327660

Gelman, A., \& Hill, J. (2007). Data analysis using regression and multilevel/hierarchical models. Cambridge University Press.

Jackson, A. S., \& Pollock, M. L. (1978). Generalized equations for predicting body density of men. British Journal of Nutrition, 40(3), 497-504. https://doi. org/10.1079/BJN19780152

Jaspers, A., Brink, M. S., Probst, S. G. M., Frencken, W. G. P., \& Helsen, W. (2017). Relationships between training load indicators and training outcomes in professional soccer. Sports Medicine, 47, 533-544. https://doi.org/10.1007/ $\underline{\text { s40279-016-0591-0 }}$

Kim, J.-H., Roberge, R., Powell, J. B., Shafer, A. B., \& Williams, W. J. (2013). Measurement accuracy of heart rate and respiratory rate during graded exercise and sustained exercise in the heat using the Zephyr BioHarness. International Journal of Sports Medicine, 34(6), 497-501. https://doi.org/10.1055/s-0032-1327661

Krustrup, P., Mohr, M., Amstrup, T., Rysgaard, T., Johansen, J., Steensberg, J., Pedersen, P. K., \& Bangsbo, J. (2003). The Yo-Yo intermittent recovery test: Physiological response, reliability, and validity. Medicine \& Science in Sports \& Exercise, 35(4), 697-705. https://doi.org/10.1249/01.MSS.0000058441.94520.32 Krustrup, P., Mohr, M., Ellingsgaard, H., \& Bangsbo, J. (2005) Physical demands of elite female soccer games: Importance of training status. Medicine \& Science in Sports \& Exercise, 37(7), 1242-1248. https://doi.org/10.1249/01. mss.0000170062.73981.94

McGilchrist, C. A., \& Yau, K. K. W. (2007). The derivation of BLUP, ML, REML estimation methods for generalized linear mixed models. Communications in Statistics - Theory and Methods, 24(12), 2963-2980. https://doi. org/10.1080/03610929508831663

Mohr, M., Krustrup, P. \& Bangsbo, J. (2003). Match performance of high-standard soccer players with special reference to development of fatigue. Journal of Sports Sciences, 21(7), 519-528. https://doi.org/10.1080/0264041031000071182

Tierney, P. J., Young, A., Clarke, N. D., \& Duncan, M. J. (2016). Match play demands of 11 versus 11 professional football using Global Positioning System tracking: Variations across common playing formations. Human Movement Science, 49, 1-8. https://doi.org/10.1016/j.humov.2016.05.007

Vilamitjana, J., Heinze, G., Verde, P., \& Calleja-González, J. (2020). Comparison of physical performance between possession games and matches in professional football. Apunts. Educación Física y Deportes, 141, 75-86. https://doi. org/10.5672/apunts.2014-0983.es.(2020/3).141.09 